\title{
Intravitreal autologous plasmin as a therapeutic modality for diffuse diabetic macular edema
}

\author{
This article was published in the following Dove Press journal: \\ Clinical Ophthalmology \\ 10 December 2012 \\ Number of times this article has been viewed
}

\author{
Moataz F Elsawy \\ Ophthalmology Department, Faculty \\ of Medicine, Menofia University, \\ Menofia, Egypt
}

Objectives: To evaluate the outcome of vitreolysis using intravitreal autologous plasmin (IAP) injection as a sole therapeutic modality for patients with diffuse diabetic macular edema in comparison to triamcinolone acetonide (TA).

Patients and methods: The study included 50 diabetic patients, 18 males and 32 females, with a mean age of $66.4 \pm 7.8$ years and a mean duration of diabetes of $14 \pm 2.4$ years. All patients underwent full ophthalmologic examination and were allocated randomly into two groups: the TA group received $4 \mathrm{mg}$ TA and the IAP group received $0.2 \mathrm{~mL}$ freshly home-prepared autologous plasmin. Outcome measures included determination of central macular thickness (CMT), best-corrected visual acuity (BCVA), and intraocular pressure (IOP) at 1, 3, and 6 months.

Results: Both TA and plasmin induced significant decrease of CMT compared to baseline thickness with nonsignificant difference between both groups. The extent of decrease of CMT reached a peak at 1 month after injection and then started to decline until the sixth month, but was significantly thinner when compared to baseline thickness. However, the extent of deterioration was less evident with plasmin as the difference between follow-up measures at 3 and 6 months were nonsignificant compared to the 1-month measure and to each other. BCVA was significantly improved compared to baseline BCVA with a nonsignificant difference between both groups at 1 and 3 months, but at 6 months, mean BCVA significantly deteriorated in TA group compared to that recorded at 3 months, but not in the IAP group. At 3 and 6 months after injection, IOP was significantly lower in both groups compared to IOP at 1 month despite being significantly higher in the TA group, but IOP was nonsignificantly lower in the IAP group compared to baseline. Moreover, mean IOP estimated at 3 and 6 months was significantly higher in the TA group compared to the IAP group.

Conclusion: Vitreolysis using IAP injection provided effective diminution of CMT with improvement of BCVA and this effect was longer lasting and safer than intravitreal injection of TA without concomitant increase of IOP.

Keywords: intravitreal injection, autologous plasmin, triamcinolone, diabetic macular edema

\section{Introduction}

Diabetes is a global problem with significantly increasing numbers of diabetics worldwide. The diabetic population is expected to double by 2030 , which will have a tremendous impact on the prevalence of diabetic complications. ${ }^{1}$ Diabetic retinopathy is an important microvascular complication and is the leading cause of blindness in the working-age population (20-64 years old) of developed countries, and is the cause of approximately $12 \%$ of new cases of blindness annually. It is estimated that around $40 \%$ of people with diabetes aged over 40 years have some degree of retinopathy that includes approximately $8.2 \%$ with vision-threatening retinopathy. ${ }^{2-3}$
Correspondence: Moataz F Elsawy Ophthalmology Department, Faculty of Medicine, Menofia University, Egypt Tel +20 1010044245

Email mfelsawy@yahoo.co.uk 
Pathogenesis of diabetic retinopathy could be attributed to activation of multiple cellular pathways, primarily mediated by hyperglycemia inducing varied metabolic effects that may result in microvascular damage of the retina leading to vascular leakage (nonproliferative diabetic retinopathy) and ischemia-induced retinal neovascularization (proliferative diabetic retinopathy). Hyperglycemia-induced pathogenesis of diabetic retinopathy is related to four main biochemical alterations such as: (i) increased polyol pathway flux; (ii) increased advanced glycation end-product formation; (iii) activation of protein kinase $\mathrm{C}$ isoforms; and (iv) increased hexosamine pathway flux; these pathways terminate in increased oxidative stress, inflammation, and vascular dysfunction that result in eventual increased permeability, vascular occlusion, and local ischemia with concomitant upregulation of proangiogenic and inflammatory factors. ${ }^{4-6}$

Tissue plasminogen activators and urokinase-plasminogen activator trigger a proteinase cascade that results in the generation of high local concentrations of plasmin. Plasmin regulates angiogenesis directly by degrading matrix molecules and indirectly by activating extracellular matrix metalloproteinases. This increase in proteolytic activity permits endothelial cell degradation and generates extracellular matrix degradation products that are chemotactic. ${ }^{7-9}$

Normally, in the absence of posterior vitreous detachment, the vitreous cortex is adhered to the internal limiting lamina of the inner retina. This junction is thought to participate in the pathophysiology of diverse retinal diseases, including proliferative diabetic retinopathy and diabetic macular edema, so vitrectomy has been associated with decrease of macular edema and improvement of visual acuity in eyes of diabetic patients. Thus, many pharmacologic agents have been studied with the aim of inducing a posterior vitreous detachment in order to facilitate the surgical procedure and reduce complications of vitrectomy. ${ }^{10}$ The term "pharmacologic vitreolysis' refers to the use of enzymes to liquefy the vitreous gel, and to induce posterior vitreous detachment. Intravitreal ovine hyaluronidase injection was effective in clearing vitreous hemorrhage. ${ }^{11}$

This prospective comparative study aimed to evaluate the outcome of vitreolysis using intravitreal autologous plasmin (IAP) injection as a therapeutic modality for patients with diabetic macular edema in comparison to triamcinolone acetonide (TA).

\section{Patients and methods}

The present study was conducted at Eye Subspecialty Center, Cairo, Egypt from June 2010 until January 2012 and included 50 patients with bilateral diabetic diffuse macular edema.
Diffuse macular edema was defined using biomicroscopy and $90 \mathrm{D}$ lens with widespread leaking around the macular area under fluorescein angiography. Inclusion limit for central macular thickness (CMT) was $>360 \mu \mathrm{m}$ (normal $\geq 200 \mu \mathrm{m}$ ) confirmed by optical coherence tomography (Stratus OCT-3; Carl Zeiss Meditec, Inc, Dublin, CA, USA). ${ }^{12}$

Exclusion criteria included uncontrolled diabetes as judged by estimation of glycosylated hemoglobin level with $<9.5 \%$ as an acceptable limit for inclusion. Patients with uncontrolled hypertension or chronic renal failure were excluded. Ocular exclusion criteria included history of ocular hypertension, glaucoma, signs of ischemia in the macular area or around the retinal periphery under fluorescein angiography, eye surgery during the previous 6 months, previous posterior vitreous detachment, diagnosed by means of biomicroscopy and $+90 \mathrm{D}$ lens and/or optical coherence tomography.

After approval of the study protocol, by the Local Ethical Committee according to World Medical Association Declaration of Helsinki, 2008, and obtaining written, fully informed consent from all patients, all patients underwent full ophthalmologic exploration including slit-lamp exploration, applanation tonometry, indirect ophthalmoscopy, macular scan by means of optical coherence tomography, fundus photograph of the macular area, and fluorescein angiography. In all cases, the optic coherence tomography and biomicroscopy discarded a vitreous detachment and confirmed adherence of the vitreous cortex in the macular area. Baseline intraocular pressure (IOP) was measured by Goldmann applanation tonometry and best-corrected visual acuity (BCVA) was estimated and expressed as logMAR for comparison during follow-up. Patients were randomly allocated into two groups according to type of intravitreal injection: the TA group received $4 \mathrm{mg}$ TA in $0.1 \mathrm{~mL}$, and the IAP group received $0.2 \mathrm{~mL}$ homeprepared IAP.

\section{Preparation of autologous plasmin}

Plasmin was prepared immediately prior to injection. A blood sample was obtained from a peripheral vein and centrifuged at $4000 \mathrm{rpm}$ for 15 minutes. The resultant plasma was transferred to a vial containing streptokinase (Streptase ${ }^{\circledR}$; ZLB Behrig Laboratories, Melbourne, Australia), previously incubated for 15 minutes at $37^{\circ} \mathrm{C}$. The diluted streptokinase was then combined with plasma by vigorously shaking the vial for 5 minutes. The resulting solution was incubated again for an additional 15 minutes at $37^{\circ} \mathrm{C}$. Finally, the solution was sterilized using a $0.22 \mathrm{~mm}$ Millipore (Billerica, MA) filter, and ready to be injected. ${ }^{13}$ 


\section{Injection technique}

Topical anesthesia using $1 \%$ benoxinate eye drops was performed at least three times, followed by conjunctiva washing with povidone solution and paracentesis of anterior chamber with a $27 \mathrm{G}$ needle, to avoid the posterior reflux on carrying out the intravitreal injection. Then, an intravitreal injection of $0.2 \mathrm{~mL}$ of the autologous plasmin solution (IAP group) or $4 \mathrm{mg} / 0.1 \mathrm{~mL}$ triamcinolone (TA group) was carried out with a $30 \mathrm{G}$ needle, at 3.5 or $4 \mathrm{~mm}$ of the limbus depending on whether the patient was pseudophakic or phakic, respectively. An absorbing sponge was applied at the injection point to avoid reflux. Ciprofloxacin and dexamethasone eye drops four times daily were prescribed for 5 consecutive days. A sole injection of plasmin in the eye was carried out independently of the initial response.

\section{Outcome points}

The outcome points to be recorded throughout the current study included the CMT, BCVA, and IOP; these points were determined 1,3, and 6 months after injection.

\section{Statistical analysis}

The obtained data were expressed as mean \pm standard deviation, ranges, numbers, percentages and ratios. Results were compared using the Wilcoxon rank-sum test (Z-test) for unrelated data. Statistical analysis was conducted using the SPSS statistical software (Version 15; IBM, Armonk, NY). A $P$ value $<0.05$ was considered statistically significant.

\section{Results}

The study included 50 patients, 18 males and 32 females, with a mean age of $66.4 \pm 7.8$ years (range, 45-79 years). All patients were diabetics with a mean duration of diabetes of $14 \pm 2.4$ years (range, 9-19 years) and a mean glycosylated hemoglobin level of $6.8 \pm 1.2 \mathrm{mmHg}$ (range, $3.9 \%-9 \%$ ). Seventeen patients had associated comorbidities: 10 patients were dyslipidemic, four patients were hypertensives, and three patients were cardiac. Thirty-one patients had affection of right eye, while 19 patients had affection of left eye. Six patients were pseudophakic, while the other 44 patients were phakic. There was nonsignificant difference between both study groups with respect to the enrollment data (Table 1).

Both TA and plasmin induced significant $(P<0.05)$ decrease of CMT compared to baseline thickness. The extent of decrease of CMT reached a peak at 1 month after injection and then started to decline till the sixth month after injection, but was still significantly $(P<0.05)$ thinner compared

Table I Patient enrollment data

\begin{tabular}{|c|c|c|c|}
\hline & TA group & IAP group & Total \\
\hline Age (years) & $65.4 \pm 8.5(45-77)$ & $67.4 \pm 7$ (49-79) & $66.4 \pm 7.8(45-79)$ \\
\hline \multicolumn{4}{|l|}{ Gender } \\
\hline Males & $8(32 \%)$ & $10(40 \%)$ & $18(36 \%)$ \\
\hline Females & 17 (68\%) & $15(60 \%)$ & $32(64 \%)$ \\
\hline \multicolumn{4}{|l|}{ Diabetes data } \\
\hline Duration of DM (years) & $14.3 \pm 2.5(9-19)$ & $13.6 \pm 2.3(11-18)$ & $14 \pm 2.4(9-19)$ \\
\hline Glycosylated hemoglobin (\%) & $6.6 \pm 1.3(3.9-8.5)$ & $7 \pm 1 . I(4.9-9)$ & $6.8 \pm 1.2(3.9-9)$ \\
\hline \multicolumn{4}{|l|}{ Associated comorbidities } \\
\hline None & 18 (72\%) & $15(60 \%)$ & $33(66 \%)$ \\
\hline Dyslipidemia & $4(16 \%)$ & $6(24 \%)$ & $10(20 \%)$ \\
\hline Hypertension & $2(8 \%)$ & $2(8 \%)$ & $4(8 \%)$ \\
\hline Cardiac & $\mathrm{I}(4 \%)$ & $2(8 \%)$ & $3(6 \%)$ \\
\hline \multicolumn{4}{|l|}{ Local examination } \\
\hline \multicolumn{4}{|l|}{ Side } \\
\hline Right & $16(64 \%)$ & $15(60 \%)$ & 31 (62\%) \\
\hline Left & $9(36 \%)$ & $10(40 \%)$ & $19(38 \%)$ \\
\hline \multicolumn{4}{|l|}{ Lens } \\
\hline Phakic & $21(84 \%)$ & $23(92 \%)$ & $44(88 \%)$ \\
\hline Pseudophakic & $4(16 \%)$ & $2(8 \%)$ & $6(12 \%)$ \\
\hline \multicolumn{4}{|l|}{ Previous treatment } \\
\hline Bevacizumab & $4(16 \%)$ & $5(20 \%)$ & $9(18 \%)$ \\
\hline Bevacizumab + TA & $7(28 \%)$ & $5(20 \%)$ & $12(24 \%)$ \\
\hline $\mathrm{PC}+\mathrm{TA}$ & $9(36 \%)$ & II (44\%) & $20(40 \%)$ \\
\hline $\mathrm{PC}+\mathrm{TA}+$ bevacizumab & $5(20 \%)$ & $4(16 \%)$ & $9(18 \%)$ \\
\hline
\end{tabular}

Note: Data are presented as mean \pm standard deviation with ranges and percentages in parenthesis.

Abbreviations: DM, diabetes mellitus; PC, photocoagulation; TA, triamcinolone acetonide. 
to baseline CMT. However, the extent of deterioration was more evident with TA and was manifested as significantly $(P<0.05)$ thicker macula at 6 months compared to the estimated thickness at 1 and 3 months with significantly $(P<0.05)$ thicker macular at 3 months compared to the thickness at 1 month. On contrary, the extent of deterioration was less evident with plasmin as the difference between follow-up measures at 3 and 6 months were nonsignificant $(P>0.05)$ compared to the measures at 1 month and to each other. Despite the nonsignificant $(P>0.05)$ difference in CMT between both TA and plasmin at 1 and 3 months, the difference at 6 months was significantly $(P<0.05)$ in favor of plasmin (Table 2 and Figure 1).

Intravitreal therapy, irrespective of medication used, induced improvement of BCVA with a nonsignificant difference $(P>0.05)$ between both groups. In comparison to baseline measures, improvement was significantly $(P<0.05)$ evident at 1 month after injection and started to decline thereafter to be nonsignificantly $(P>0.05)$ better compared to baseline measures at 6 months after injection. Mean BCVA significantly $(P<0.05)$ deteriorated in the TA group at 6 months compared to 3 months, however, in the IAP group, the difference was nonsignificant $(P>0.05)$ despite the deterioration (Table 2 and Figure 2).

Intravitreal therapy, irrespective of medication used, induced significant elevation of IOP at 1 month after injection with significantly lower IOP in the IAP group compared to the TA group. At 3 and 6 months after injection, IOP was significantly lower in both groups compared to the IOP at 1 month despite being significantly higher compared to baseline IOP in the TA group, but was nonsignificantly lower in IAP group compared to baseline IOP. Moreover, the mean IOP estimated at 3 and 6 months was significantly higher in the TA group compared to the IAP group (Table 2 and Figure 3).

\section{Discussion}

Intravitreal therapy, irrespective of medication used, induced significant improvement as regards CMT and BCVA and this indicated the beneficial effect of such therapeutic modality for management of diffuse diabetic macular edema (DDME). In line with the obtained results; Senturk et a ${ }^{14}$ reported that in eyes with macular edema in central retinal vein occlusion, a short-term improvement in retinal sensitivity and fixation properties was achieved by intravitreal TA injection. Khurieva-Sattler et al ${ }^{15}$ evaluated visual outcome and anatomic results in patients with DDME after vitrectomy with internal limiting membrane peeling versus intravitreal TA and found intravitreal TA as a single treatment reduces the extent of DDME within a short time after surgery, but these promising results may not be stable during long-term follow-up, necessitating a reinjection of TA in many cases. These previous studies in line with current study stressed on the short duration of the effect of intravitreal TA injection.

In support of the beneficial effect of intravitreal injection of autologous plasmin enzyme (APE), Díaz-Llopis et al ${ }^{12,16}$ tried to determine the effectiveness of a low-dose intravitreal injection of APE without vitrectomy as a treatment for refractory DDME and reported that intravitreal APE injection effectively reduced macular thickening due to DDME in cases that fail to respond to conventional laser photocoagulation, and improves visual acuity in the short term, and importantly, this result remains stable in the medium term. Sakuma et $\mathrm{al}^{17}$ and Udaondo et $\mathrm{al}^{18}$ evaluated the effects and safety of intravitreal injections of APE, without vitrectomy, as a treatment for macular edema secondary to branch retinal vein occlusion and reported that IAP improves CMT and BCVA and may be a safe and effective alternative therapy for this condition.

The extent of improvement was more pronounced with IAP compared to TA as manifested by the significantly less

Table 2 Postinjection data compared to baseline data

\begin{tabular}{|c|c|c|c|c|}
\hline & Baseline & I month & 3 month & 6 month \\
\hline \multicolumn{5}{|c|}{$\mathrm{CMT}(\mu \mathrm{m})$} \\
\hline TA & $539.4 \pm 85.2$ & $302 \pm 42.6^{*}$ & $319.3 \pm 50^{*, \dagger}$ & $337 \pm 57.3^{*,+, \#}$ \\
\hline IAP & $542.5 \pm 90.2$ & $304.2 \pm 46.8^{*}$ & $316 \pm 44.2^{*}$ & $322 \pm 57.3^{*, \neq}$ \\
\hline \multicolumn{5}{|c|}{ BCVA (logMAR) } \\
\hline TA & $0.412 \pm 0.09$ & $0.24 \pm 0.12 *$ & $0.312 \pm 0.129 *,+$ & $0.386 \pm 0.093^{*,+, \neq}$ \\
\hline IAP & $0.402 \pm 0.095$ & $0.248 \pm 0.116^{*}$ & $0.289 \pm 0.108^{*, \dagger}$ & $0.346 \pm 0.1 \mathrm{I}^{\dagger}$ \\
\hline \multicolumn{5}{|c|}{ IOP (mmHg) } \\
\hline TA & $15.8 \pm 1.8^{*}$ & $17.8 \pm 1.8^{*}$ & $17.2 \pm 1.7^{*, \dagger}$ & $16.9 \pm 2^{*, \dagger}$ \\
\hline IAP & $15.7 \pm 1.9$ & $16 \pm 1.9 * \#$ & $15.7 \pm 1.8^{\dagger, \#}$ & $15.4 \pm 2^{\dagger, \#}$ \\
\hline
\end{tabular}

Notes: Data are presented as mean \pm standard deviation and ranges and percentages are in parenthesis. ${ }^{2}$ Significant versus baseline measures; ${ }^{\dagger}$ significant versus I-month

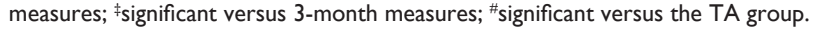

Abbreviations: BCVA, best-corrected visual acuity; CMT, central mascular thickness; IAP, intravitreal autologous plasmin; IOP, intraocular pressure; TA, triamcinolone acetonide. 


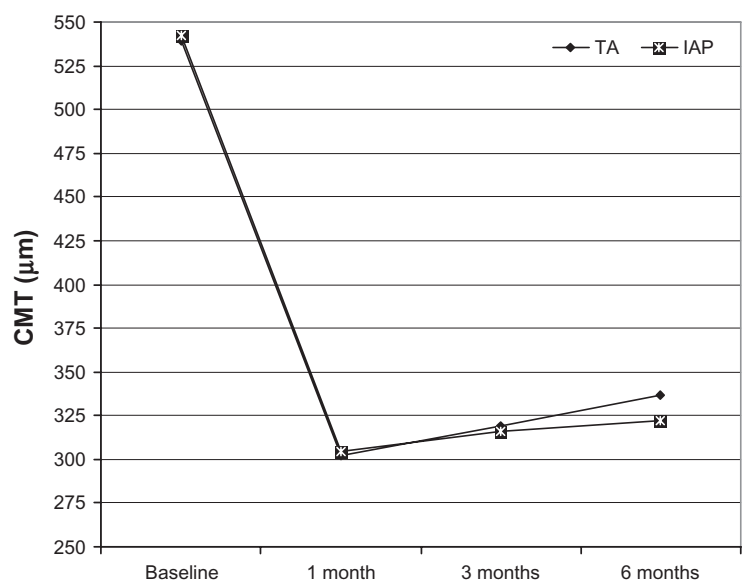

Figure I Mean CMT of eyes throughout the study period.

Abbreviations: CMT, central macular thickness; IAP, intravitreal analogous plasmin; TA, triamcinolone acetonide.

CMT estimated at 6 months with IAP compared to TA, a finding indicating a more sustained effect of IAP compared to TA and could be attributed the difference in the mechanism of action. Triamcinolone, by its virtue as a corticosteroid, acts as an anti-inflammatory and antiedematous drug through stabilization of the blood-retinal barrier and downregulation of the production of vascular endothelial growth factor. This known vascular permeability factor reduced the vascular permeability, which reduced edema and inflammation. ${ }^{19}$ However, edema and activation of inflammatory cascade are secondary events to the main pathogenic mechanism, which is the retinal hypoxia that triggers the release of reactive oxygen species and proinflammatory cytokines aggravating the condition. ${ }^{4,20}$ Once the blunting effect of the corticosteroid fades away, the condition recurs and this could explain the short-lived effect of intravitreal TA.

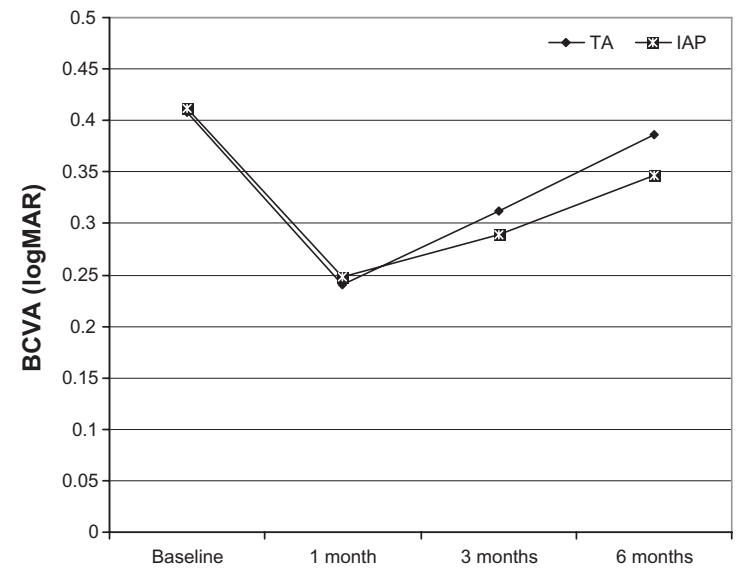

Figure 2 Mean BCVA of both groups throughout the study period. Abbreviations: BCVA, best-corrected visual acuity; IAP, intravitreal analogous plasmin; TA, triamcinolone acetonide.

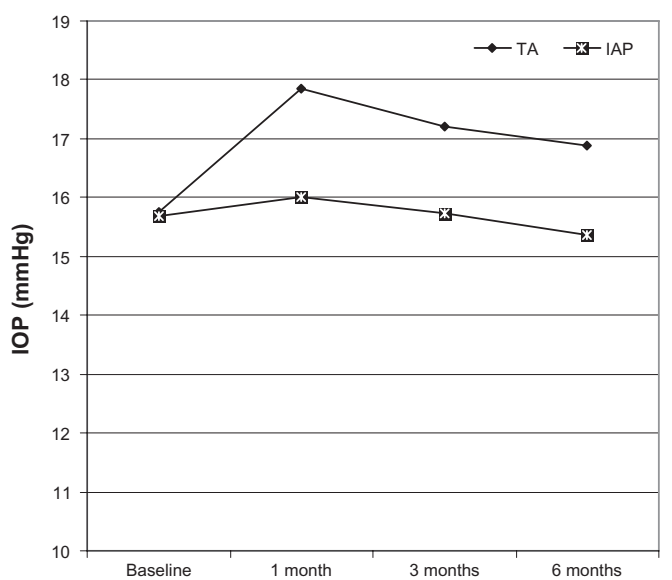

Figure 3 Mean IOP estimated in both groups throughout the study period. Abbreviations: IAP, intravitreal analogous plasmin; IOP, intraocular pressure; TA, triamcinolone acetonide.

In support of such attribution, Boyer et $\mathrm{al}^{21}$ found treatment with dexamethasone intravitreal implant led to statistically and clinically significant improvements in both vision and vascular leakage from diabetic macular edema in difficultto-treat vitrectomized eyes and had an acceptable safety profile. Moreover, Elbendary and Shahin ${ }^{22}$ found intravitreal diclofenac is effective in the treatment of DDME up to 12 weeks and intravitreal triamcinolone and diclofenac appear to have comparable therapeutic effects on retinal thickness.

The proteolytic activities of an intravitreal plasmin injection breaks the adhesion between retina and posterior vitreous, which induces posterior retinal detachment, and relieves retinal hypoxia with subsequent events that hamper rapid recurrence with pronounced and sustained effects till 6 months after injection. Gandorfer ${ }^{23}$ showed that pharmacologic vitreolysis cleaves the vitreoretinal junction, cleaves the cortical hyaloid completely from the retina, changes the molecular flux across the vitreoretinal interface, and improves oxygen supply to the retina, which is a major mechanism of action that might significantly interfere with biochemical pathways of retinal hypoxia, leading to an overexpression of vasoactive substances such as vascular endothelial growth factor.

Interestingly, the current study reported nonsignificant changes in IOP in the IAP group at 3 and 6 months after injection compared to their baseline IOP with significantly lower pressure compared to the TA group, which showed significantly higher IOP compared to their baseline IOP throughout the follow-up period. Elevated IOP with intravitreal TA is one of the unwanted effects of this therapeutic modality. Kramar et a ${ }^{24}$ reported that intravitreal TA is frequently associated with a significant elevation in IOP, typically within the first 2 months after injection; most patients who do not have an elevated IOP after an initial 
injection will not experience a pressure rise after an additional one and about one-third will require topical glaucoma therapy for IOP control. García Fernández et a ${ }^{25}$ reported increased IOP in $57.69 \%, 75 \%$, and $47 \%$ of patients after intravitreal TA injection at 1,3 , and 6 months, respectively, and concluded that intravitreal TA is a good therapeutic option for patients with persistent diabetic macular edema, by increasing BCVA and decreasing CMT in the short term. However, due to the transient effect and potential adverse effects, it should be administered to selected refractory cases with caution. Bozkurt et $\mathrm{al}^{26}$ evaluated the prophylactic efficacy of selective laser trabeculoplasty for preventing IOP increase after intravitreal TA injection and documented that IOP elevation after intravitreal TA injection may be prevented by performing selective laser trabeculoplasty before injection, especially in cases with a baseline IOP of $\geq 21 \mathrm{mmHg}$.

It could be concluded that vitreolysis using IAP injection provided effective diminution of CMT with improvement of BCVA and this effect was longer lasting and safer than intravitreal injection of TA without concomitant increase of IOP. However, wider scale studies for a longer duration of follow-up are mandatory to define the maximum duration of action and thus the need for repeated injection to maintain the achieved improvement.

\section{Disclosure}

The authors report no conflicts of interest in this work.

\section{References}

1. Shaw JE, Sicree RA, Zimmet PZ. Global estimates of the prevalence of diabetes for 2010 and 2030. Diabetes Res Clin Pract. 2010;87:4-14.

2. Aiello LM. Perspectives on diabetic retinopathy. Am J Ophthalmol. 2003;136:122-135.

3. Ciulla TA, Amador AG, Zinman B. Diabetic retinopathy and diabetic macular edema: pathophysiology, screening, and novel therapies. Diabetes Care. 2003;26:2653-2664.

4. Brownlee M. The pathobiology of diabetic complications: A unifying mechanism. Diabetes. 2005;54:1615-1625.

5. Pober JS, Sessa WC. Evolving functions of endothelial cells in inflammation. Nat Rev Immunol. 2007;7:803-815.

6. Sang DN, D'Amore PA. Is blockade of vascular endothelial growth factor beneficial for all types of diabetic retinopathy? Diabetologia. 2008;51:1570-1573.

7. Mignatti P, Rifkin DB. Plasminogen activators and matrix metalloproteinases in angiogenesis. Enzyme Protein. 1996;49:117-137

8. Castellino FJ, Ploplis VA. Structure and function of the plasminogen/ plasmin system. Thromb Haemost. 2005;93:647-654.

Clinical Ophthalmology

\section{Publish your work in this journal}

Clinical Ophthalmology is an international, peer-reviewed journal covering all subspecialties within ophthalmology. Key topics include: Optometry; Visual science; Pharmacology and drug therapy in eye diseases; Basic Sciences; Primary and Secondary eye care; Patient Safety and Quality of Care Improvements. This journal is indexed on Submit your manuscript here: http://www.dovepress.com/clinical-ophthalmology-journal
9. Adibhatla RM, Hatcher JF. Tissue plasminogen activator (tPA) and matrix metalloproteinases in the pathogenesis of stroke: therapeutic strategies. CNS Neurol Disord Drug Targets. 2008;7:243-253.

10. Lopez-Lopez F, Rodriguez-Blanco M, Gómez-Ulla F, Marticorena J. Enzymatic vitreolysis. Curr Diabetes Rev. 2009;5:57-62.

11. El-Asrar AM, Al-Mezain HS. Pharmacologic vitreolysis in diabetic retinopathy. Curr Pharm Biotechnol. 2011;12:406-409.

12. Díaz-Llopis M, Udaondo P, Arevalo F, et al. Intravitreal plasmin without associated vitrectomy as a treatment for refractory diabetic macular edema. J Ocul Pharmacol Ther. 2009;25:379-384.

13. Rizzo S, Pellegrini G, Benocci F, Belting C, Baicchi M, Vispi M. Autologous plasmin for pharmacologic vitreolysis prepared 1 hour before surgery. Retina. 2006;26:792-796.

14. Senturk F, Ozdemir H, Karacorlu M, Karacorlu SA, Uysal O. Microperimetric changes after intravitreal triamcinolone acetonide injection for macular edema due to central retinal vein occlusion. Retina. 2010;30:1254-1261.

15. Khurieva-Sattler E, Krause M, Löw U, et al. Comparison of pars plana vitrectomy with ILM peeling and intravitreal triamcinolone in diffuse diabetic macular edema. Klin Monbl Augenheilkd. 2010;227:496-500.

16. Díaz-Llopis M, Udaondo P, García-Delpech S, Cervera E, Salom D, Quijada A. Enzymatic vitrectomy by intravitreal autologous plasmin injection, as initial treatment for diffuse diabetic macular edema. Arch Soc Esp Oftalmol. 2008;83:77-84.

17. Sakuma T, Mizota A, Inoue J, Tanaka M. Intravitreal injection of autologous plasmin enzyme for macular edema associated with branch retinal vein occlusion. Am J Ophthalmol. 2010;150:876-882.

18. Udaondo P, Díaz-Llopis M, García-Delpech S, Salom D, Romero FJ. Intravitreal plasmin without vitrectomy for macular edema secondary to branch retinal vein occlusion. Arch Ophthalmol. 2011;129:283-287.

19. Wilson CA, Berkowitz BA, Sato Y, Ando N, Handa JT, de Juan E Jr: Treatment with intravitreal steroid reduced blood-retinal barrier breakdown due to retinal photocoagulation. Arch Ophthamol. 1992; 110(8):1155-1159.

20. Rangasamy S, McGuire PG, Das A. Diabetic retinopathy and inflammation: novel therapeutic targets. Middle East Afr J Ophthalmol. 2012; 19:52-59.

21. Boyer DS, Faber D, Gupta S, et al; Ozurdex CHAMPLAIN Study Group. Dexamethasone intravitreal implant for treatment of diabetic macular edema in vitrectomized patients. Retina. 2011;31:915-923.

22. Elbendary AM, Shahin MM. Intravitreal diclofenac versus intravitreal triamcinolone acetonide in the treatment of diabetic macular edema. Retina. 2011;31:2058-2064.

23. Gandorfer A. Objective of pharmacologic vitreolysis. Dev Ophthalmol. 2009;44:1-6.

24. Kramar M, Vu L, Whitson JT, He YG. The effect of intravitreal triamcinolone on intraocular pressure. Curr Med Res Opin. 2007;23: 1253-1258.

25. García Fernández M, García Alonso A, Fonollá Gil M, Rodríguez Villa S. Intravitreal triamcinolone acetonide use in diffuse persistent diabetic macular edema. Arch Soc Esp Oftalmol. 2011;86:314-319.

26. Bozkurt E, Kara N, Yazici AT, et al. Prophylactic selective laser trabeculoplasty in the prevention of intraocular pressure elevation after intravitreal triamcinolone acetonide injection. Am J Ophthalmol. 2011;152:976-981.

\section{Dovepress}

PubMed Central and CAS, and is the official journal of The Society of Clinical Ophthalmology (SCO). The manuscript management system is completely online and includes a very quick and fair peer-review system, which is all easy to use. Visit http://www.dovepress.com/ testimonials.php to read real quotes from published authors. 